\title{
Community Health Workers: A Missing Piece of the Puzzle for Complex Patients With Diabetes?
}

\author{
Maria E. Garcia, MD MPH ${ }^{1,3}$ and Richard W. Grant, MD MPH ${ }^{2}$ \\ 'Division of General Internal Medicine, San Francisco, CA, USA; ${ }^{2}$ Division of Research, Kaiser Permanente Northern California, Oakland, CA, USA; \\ ${ }^{3}$ Division of General Internal Medicine, San Francisco General Hospital, San Francisco, CA, USA.
}

J Gen Intern Med 30(7):878-9

DOI: $10.1007 / \mathrm{s} 11606-015-3320-8$

(c) Society of General Internal Medicine 2015

$\mathrm{T}$ he prevalence and incidence of type 2 diabetes in the U.S. continues to rise, particularly among racial and ethnic minorities and individuals with low socioeconomic status. The U.S. population with diabetes is also increasingly complex, due in part to a greater prevalence of comorbidities, changing demographics, and the unique challenges faced by vulnerable populations in accessing care. Many "nonmedical" factors further contribute to patient complexity, including language abilities, socioeconomic barriers, financial stressors, and inadequate insurance coverage. ${ }^{1}$ And with the advent of the Affordable Care Act, more Latinos and other chronically uninsured or under-insured patients are now presenting for primary care ${ }^{2}$ at a time of continuing shortage of culturally and linguistically concordant primary care providers. $^{3}$

Effective diabetes care requires coordination among various providers, significant lifestyle modifications, and adherence to multiple concurrent medications. ${ }^{4}$ As a greater number of medically and socially complex patients with diabetes are seen in primary care, providers struggle with how to address numerous treatment guidelines, referral and screening requirements, and patient education needs in shorter amounts of time. ${ }^{5}$ Achieving evidence-based standards of care for patients with diabetes remains an unsolved challenge, with nearly onehalf of adults with type 2 diabetes not meeting all evidencebased recommendations for diabetes care. ${ }^{6}$ Furthermore, an increasing comorbidity burden and the aging of the primary care population are placing a significant strain on our primary health care system. Indeed, one in four adults in the U.S. has multiple concurrent chronic conditions, and the treatment of these individuals accounts for over $80 \%$ of Medicare expenditures. ${ }^{7}$ Thus, new models of primary care are needed.

In this issue of JGIM, Palmas et al. review the current evidence supporting the role of community health workers (CHWs) in addressing gaps in diabetes care. ${ }^{8} \mathrm{CHWs}$ are generally lay personnel with varying levels of medical training who have expertise in local community resources and

Published online April 11, 2015 often share cultural and socioeconomic characteristics with their assigned patients. Programs involving CHWs, or Promotoras and Promotores de Salud in Spanish, have been implemented in a variety of care settings and have focused on a variety of different health conditions. Palmas et al. performed a systematic review of nine U.S. randomized controlled trials (13 trials in the narrative review) that implemented CHW interventions among ethnic and racial minorities (primarily Latinos and African Americans) and individuals from low socioeconomic status with type 2 diabetes. Despite widely varying study designs, a small overall number of participants, and differing levels of CHW training among the trials, a significant, if modest, improvement in glycemic control was found with CHW intervention (with a standardized mean difference in A1c of $0.21,95 \%$ CI $0.11,0.32$ ). As the authors note, this effect size is similar in magnitude to benefits associated with psychosocial interventions and with implementation of the Chronic Care Model to diabetes care. ${ }^{9,10}$

CHW programs may improve diabetes control among medically underserved individuals through a number of different mechanisms: CHWs may help to advocate for patients, reduce cultural barriers to care, improve communication within different facets of the health care system, and/or navigate patients through complicated care systems. Some CHWs may also provide needed health education and convey a personal investment in the patient. Given these myriad roles, current clinically oriented outcomes such as hemoglobin A1c may not be adequately measuring many of the beneficial aspects of CHWs. Future studies should also examine the impact of CHWs on domains such as patient engagement, self-management, and satisfaction with care.

The review by Palmas et al. provides support for the implementation of CHWs as a way to engage certain medically underserved populations. However, important questions regarding the expansion of CHWs remain. For one, approaches to CHW program implementation are not standardized. In particular, the optimal training (both in content and duration) for new CHWs is not well defined. In addition, these programs are often not well integrated into existing care systems. In most of the studies cited by Palmas et al, for example, CHW programs were run by independent non-profit organizations rather than by the medical practices themselves. CHW programs also vary in the amount of interaction, if any, between 
CHWs and members of the clinical team (including nurses, social workers, and primary care providers). There are few mechanisms currently in place for providers to bill for time spent supporting CHWs in the care of patients. Policy changes at the system level are needed to more effectively integrate these personnel into the care team. One challenge to the successful implementation of CHW programs, for example, will be to understand how CHW programs fit into new payment structures mandated by the ACA.

The systematic review only included studies with a 12 month follow-up period. The minimum time frame for CHW intervention programs remains ill-defined, and it is unknown whether the benefits from such interventions are sustained. Part of the difficulty in implementing and evaluating CHW programs is the need to balance standardized intervention components with the flexibility necessary for CHWs to respond to the varying needs of individual patients.

Since most CHW programs have been implemented in clinics serving racial and ethnic minorities (primarily Latinos and African Americans, with only one study each including Samoans and whites) and/or those with low socioeconomic status, we also do not yet know whether this model can be applied to other patient populations such as older patients or patients with lower literacy and numeracy skills from all race/ ethnic groups.

Future research must also evaluate CHWs in the context of other programs designed to individualize care and reach vulnerable and medically underserved populations. These include peer health coaches, diabetes educators co-located in primary care clinics, and team-based approaches that rely on shared responsibility among pharmacists, medical assistants, nurses, and physicians for achieving evidence-based diabetes care. Given the frequent co-diagnosis of mental health conditions with diabetes, and worse outcomes among these patients, other programs have also incorporated behavioral interventions to address mental health and self-management skills in order to increase patient engagement and motivation. Optimal application and wider dissemination of $\mathrm{CHW}$ programs will require successful integration and collaboration with existing programs and care team models created to support patients with diabetes.

The delivery of medical care must improve on many fronts to reduce existing barriers to patients receiving optimal management. As diabetes continues to expand in vulnerable populations, care systems will need to integrate evidence-based diabetes treatment guidelines with tools to address cultural, economic, and socioeconomic barriers to effective selfmanagement and care engagement. CHWs may represent a key tool for reducing disparities in diabetes care by reaching populations from different cultural backgrounds that may require a more human touch or personalized approach. However, it is worth emphasizing that CHWs provide value to a large extent because of the limitations of our current care system. CHWs can be seen as a critical first step to improving the experience of vulnerable populations, but real progress will be made only when barriers faced by all patients interfacing with our current health system are more effectively addressed.

Acknowledgments: R.W.G receives support from the National Institute of Diabetes and Digestive and Kidney Diseases (NIDDK; R01DK099108), the National Heart, Lung, and Blood Institute (NHLBI; RO1 HL117939), and the Patient-Centered Outcomes Research Institute (PCORI; SC14-1403-11992).

Corresponding Author: Maria E. Garcia, MD MPH; Division of General Internal MedicineSan Francisco General Hospital, 1001 Potrero Ave. $1 \mathrm{M3}$, San Francisco, CA 94110, USA (e-mail: Maria.Garcia@ucsf.edu).

\section{REFERENCES}

1. Piette JD, Kerr EA. The impact of comorbid chronic conditions on diabetes care. Diabetes Care. 2006;29:725-31.

2. Doty MM, Blumenthal D, Collins SR. The affordable care act and health insurance for Latinos. JAMA. 2014;312:1735-6.

3. Brotherton SE, Etzel SI. Graduate medical education, 2010-2011. JAMA. 2011:306:1015-30.

4. Standards of medical care in diabetes-2015. Summary of revisions. Diabetes care 2015;38 Suppl:S4. doi:10.2337/dc15-S003

5. Chaudhry SI, Berlowitz DR, Concato J. Do age and comorbidity affect intensity of pharmacological therapy for poorly controlled diabetes mellitus? J Am Geriatr Soc. 2005;53:1214-6.

6. Ali MK, Bullard KM, Saaddine JB, Cowie CC, Imperatore G, Gregg EW. Achievement of goals in U.S. diabetes care, 1999-2010. N Engl J Med. 2013;368: 1613-24.

7. Chronic Conditions among Medicare Beneficiaries, Chartbook, 2012 Edition. Centers for Medicare and Medicaid Services, 2012. (Accessed at http://www. cms.gov/Research-Statistics-Data-and-Systems/Statistics-Trends-andReports/Chronic-Conditions/Downloads/2012Chartbook.pdf.)

8. Palmas W, March D, Darakjy S, Findley SE, Teresi J, Carrasquillo O, Luchsinger JA. Community Health Worker Interventions to Improve Glycemic Control in people with Diabetes: A Systematic Review and Meta-Analysis. J Gen Intern Med. 3247. doi:10.1007/s11606-015-3247-0

9. Harkness E, Macdonald W, Valderas J, Coventry P, Gask L, Bower P. Identifying psychosocial interventions that improve both physical and mental health in patients with diabetes: a systematic review and metaanalysis. Diabetes Care. 2010;33:926-30.

10. Tsai AC, Morton SC, Mangione CM, Keeler EB. A meta-analysis of interventions to improve care for chronic illnesses. Am J Manag Care. 2005; 11:478-88. 